\title{
The current state of lung transplantation for cystic fibrosis
}

\author{
Rosalind L Smyth, Tim Higenbottam, John Scott, John Wallwork
}

As survival in cystic fibrosis has improved over the last decade, ${ }^{1}$ new treatment for the terminal pulmonary complications has become available. The first successful heart-lung transplantations for cystic fibrosis were performed in $1985^{2}$ and early results were excellent in five of the first six patients to receive a transplant. ${ }^{3}$ Subsequently over 100 patients worldwide have received transplants. As more physicians caring for patients with advanced lung disease and patients themselves have become more aware of transplantation, the demand for these scarce resources has increased substantially. There is a need for careful selection of patients and for surgical, postoperative, and long term management by an experienced transplant team. We review the current experience of heart-lung transplantation for patients with cystic fibrosis and make recommendations for the referral of patients.

\section{Operative procedures}

Despite many attempts at lung transplantation in the 1960s and 1970s there were no long term survivors. Prolonged survival was achieved in primates in the late $1970 \mathrm{~s}^{4}$ by the use of the immunosuppressive agent cyclosporin and a combined heart and lung transplant operation. This led in 1981 to the first successful human heart-lung transplant at Stanford, California, in a patient with pulmonary vascular disease. ${ }^{5}$ The en bloc surgical procedure, which required only three straightforward anastomoses (tracheal, aortic, and right atrial), offered substantial technical advantages and achieved better healing of the tracheal anastomosis. ${ }^{5}$ This operation has required little modification for use in patients with cystic fibrosis. Spillage of purulent secretions from the bronchi and trachea are avoided and the recipient tracheal stump is washed with betadine solution before anastomosis. ${ }^{6}$ The "domino" operation, in which the recipient's heart is donated to another patient, is now routine in Britain. ${ }^{6}$ This heart almost always has some degree of right ventricular hypertrophy ${ }^{3}$ and minimal coronary atheroma and is highly suitable for transplantation, particularly for the patient with an increased pulmonary vascular resistance.

An alternative operation that is practical for patients with cystic fibrosis is a double lung transplantation. This procedure was pioneered in patients with chronic obstructive lung disease ${ }^{7}$ but has been extended to patients with cystic fibrosis. ${ }^{8}$ Donor heart and lungs are available for two separate recipients as in heart-lung transplantation with the domino procedure. There were initial hopes that those having the double lung transplantation would not develop disabling obliterative bronchiolitis, ${ }^{7}$ an important cause of late graft failure in the Stanford and Pittsburgh transplant programmes. ${ }^{910}$ Obliterative bronchiolitis has been observed, however, in long term survivors with single lung transplants ( $G$ A Patterson, personal communication). Airway complications occur rarely with heartlung transplantation, but are an important cause of morbidity and death after double lung transplantation. ${ }^{78}$ Ischaemic complications of the airways occurred in eight of the first 17 recipients of double lung transplants in the Toronto series. ${ }^{8}$ Airway necrosis caused or contributed to death in four patients and required retransplantation in one. Three other patients developed strictures. In an attempt to reduce airway ischaemia Metras et al ${ }^{11}$ have modified Cooper's technique for constructing bilateral bronchial anastomoses using single running sutures. Nine recipients of double lung transplants with cystic fibrosis received this operation. Airway complications occurred in three; one died from bilateral bronchial dehiscence and two required stents.

\section{Selection of recipients}

Assessment for transplantation aims to identify those patients likely to benefit from this treatment by improved survival and quality of life. The individual's prognosis and degree of disability must therefore be evaluated. Survival in cystic fibrosis is difficult to predict accurately. Pulmonary variables related to poor outcome include a forced expiratory volume in one second $\left(\mathrm{FEV}_{1}\right)$ less than $30 \%$ of the predicted value, ${ }^{12}$ hypoxic hypercapnic respiratory failure, and cor pulmonale. ${ }^{1314}$ One of the most important prognostic indicators is weight. A low weight percentile was an adverse prognostic indicator of five year survival in patients with cystic fibrosis at 18 years of age ${ }^{15}$ and this has been our experience also. ${ }^{16}$ These clinical variables must not be seen in isolation from the quality of life of patient and family, including ability to continue full time employment or education, frequency of hospital admissions, and requirements for physiotherapy. Many patients die while awaiting assessment and transplan- 
Time after surgery and cause of death in the five patients with cystic fibrosis who died after heart-lung transplantation in the Papworth series

\begin{aligned} & \hline Days after surgery Cause of death \\ & \hline 6 Aspiration pneumonia \\ & 11 Adult respiratory distress syndrome \\ & and cirrhosis \\ & 67 Disseminated candidial septicaemia \\ & 256 Obliterative bronchiolitis \\ & 658 Obliterative bronchiolitis \\ & \hline\end{aligned}

tation. We therefore advise physicians to refer patients with cystic fibrosis when their expectation of life is estimated to be less than two years, or when their condition can no longer be stabilised by conventional means. ${ }^{16}$

\section{Exclusion criteria}

Some patients represent an unacceptably high risk for surgery. Failure to identify such risk factors may lead to the double tragedy of an avoidable death and the loss of precious donor organs. As experience with transplantation in patients with cystic fibrosis has increased the exclusion criteria have diminished. We have now carried out transplantations, without causing additional complications, in some patients who had had a lobectomy, some with insulin dependent diabetes mellitus, and others who were receiving low dose steroids or who had Aspergillus fumigatus in their sputum cultures. Patients who have undergone pleurectomy and pleurodesis are currently excluded. ${ }^{17}$ With improved methods of controlling perioperative and postoperative bleeding, including the use of aprotinin, this may soon no longer be the case. Advanced cirrhosis, diagnosed only at necropsy, has been responsible for one death in our series ${ }^{3}$ (table). Before operation this patient had a normal prothrombin time but increased alkaline phosphatase activity. After operation she

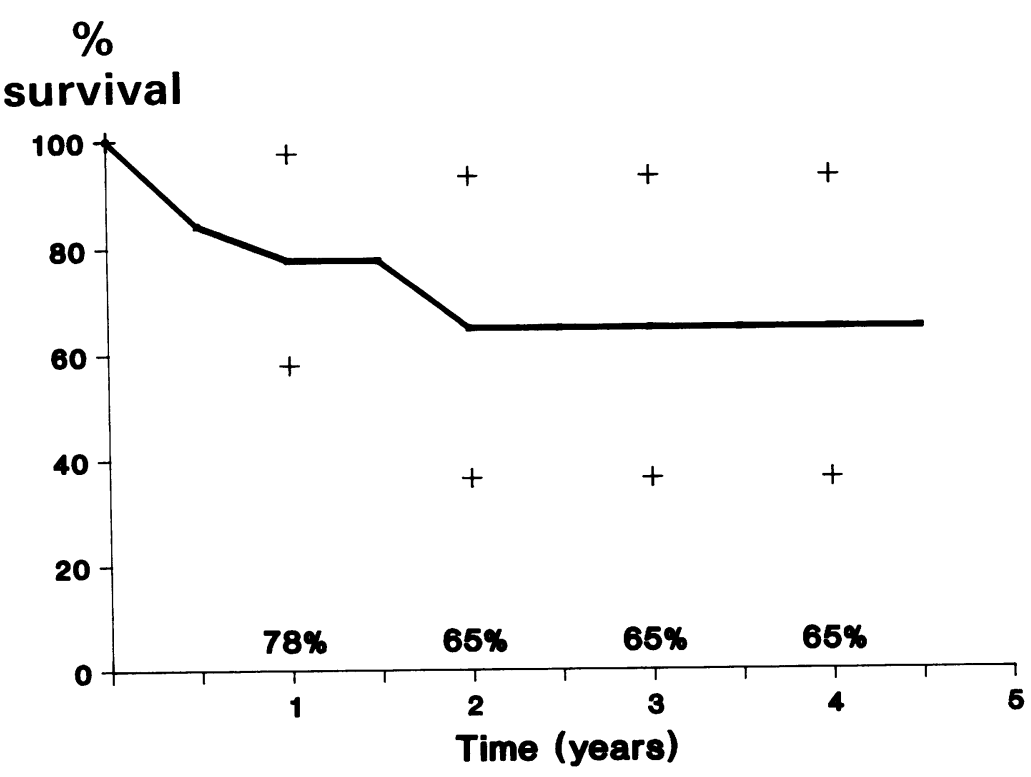

Actuarial probability of survival for the first 22 patients with cystic fibrosis who received heart-lung transplants at Papworth. + indicates standard error. developed coagulopathy, required re-exploration for bleeding, developed adult respiratory distress syndrome, and died 13 days after transplantation. This case illustrates the difficulty of identifying liver disease in patients with cystic fibrosis. Results of liver function tests may remain normal in the presence of cirrhosis and the histological changes of cirrhosis are patchy and often missed by liver biopsy. ${ }^{18}$ Portal hypertension is an unequivocal contraindication to heart-lung transplantation alone, though such patients may be suitable for heart, lung, and liver transplantation..$^{19}$ In doubtful cases evaluation by ultrasound scanning, biopsy, or hepatobiliary scintigraphy (or a combination of these) may be helpful. ${ }^{18}$ Infections that will not be eradicated by surgery or pose an unacceptable risk to other patients are contraindicated. These include extrapulmonary aspergilloma and growth of methicillin resistant Staphylococcus aureus on sputum culture. ${ }^{3}$

\section{Results of surgery}

The largest experience of heart-lung transplantation for cystic fibrosis has been gained in Britain at Harefield Hospital and in our collaborative programme between Papworth Hospital and the Hospital for Sick Children, Great Ormond Street. The early results are encouraging. We have shown the actuarial survival to one year to be $78 \%$ (figure) for patients with cystic fibrosis, which is similar to the best figures obtained for all diagnostic groups. ${ }^{20}$ Analysis of the quality of life before and after transplantation shows considerable improvements, ${ }^{21}$ and most patients have returned to work or school. In our first 28 transplanted patients mean $\mathrm{FEV}_{1}$ rose from $19 \%$ of predicted before operation to $81 \%$ three months after transplantation, and this was sustained for the first two years of follow up.

\section{Complications}

Five patients with cystic fibrosis have died in our series of 28 patients after transplantation (table). Acute rejection, opportunist lung infection, and obliterative bronchiolitis are the major long term complications after transplantation, ${ }^{22}$ and the incidence is the same for patients with cystic fibrosis as for other diagnostic groups. ${ }^{6}$ The pathogenesis, diagnosis, and treatment of these conditions is well documented $^{22-25}$ and will not be discussed here.

Certain extrapulmonary complications are peculiar to patients with cystic fibrosis. The distal intestinal obstruction syndrome or "meconium ileus equivalent" some patients postoperatively, undoubtedly precipitated by the diuresis required at this time. ${ }^{11}{ }^{27}$ In all but two cases this has resolved within two days with conservative management. One patient developed an associated gastric dilatation and died of aspiration pneumonia (table). In another meconium ileus equivalent was complicated by a distal ileal volvulus, which required resection; this patient 
recovered. ${ }^{28}$ Prophylaxis with oral $\mathrm{N}$-acetylcysteine and lactulose is used routinely after surgery and has proved effective.

Maintenance immunosuppression is with oral cyclosporin and azothioprine. Episodes of rejection are treated with intravenous and oral corticosteroids and patients with recurrent episodes of rejection are maintained with oral corticosteroids. Patients with cystic fibrosis often require up to 5-10 times the total daily dose of cyclosporin required by other patients. Reduction of the dosing interval from 12 hourly to eight hourly permitted a reduction in the total daily dose to about one third of that used previously (an annual saving of more than $£ 3000$ a patient). ${ }^{29}$ Transient hyperglycaemia often occurs in patients with cystic fibrosis, and occasionally in other patients, without diabetes mellitus during corticosteroid treatment. All episodes have been controlled with insulin and have resolved after reduction in corticosteroid dose.

\section{Recurrence of disease}

A major concern regarding heart-lung transplantation in patients with cystic fibrosis was that the ion transport defect might develop in the donor lungs. The potential difference across the respiratory mucosa, which is increased in patients with cystic fibrosis, ${ }^{30}$ has remained normal in the transplanted lungs up to two years after transplantation, whereas the trachea above the anastomosis retains the high potential difference characteristic of cystic fibrosis. ${ }^{30} 31$ This is in keeping with the hypothesis that the genetically determined ion transport defect in cystic fibrosis is expressed at a cellular level in the respiratory epithelium and not as a result of circulating mediators. ${ }^{32}$

Another anxiety was that patients with suppurative lung disease when transplanted and immunosuppressed would develop overwhelming sepsis. After transplantation colonisation of the respiratory tract with organisms similar to those grown before surgery does occur, presumably by contamination from the upper airways. Chronic pseudomonas bronchitis was identified as a specific syndrome, however, in an early series of heart-lung transplantation in patients without cystic fibrosis. ${ }^{33}$ In Cambridge over half of the patients receiving transplants for pulmonary vascular disease have had Staphylococcus aureus or Pseudomonas species grown on sputum cultures. The number of episodes of pneumonia and bronchitis was no greater in recipients with cystic fibrosis than in this group. This supports the conclusion that the intrapulmonary problems observed in patients with cystic fibrosis after heart-lung transplantation relate to transplantation rather than to cystic fibrosis.

\section{Conclusions}

Lung transplantation is a practical treatment for the terminal pulmonary complications of cystic fibrosis. Surgical and medical management of heart-lung transplantation and double lung transplantation can be extended to patients with cystic fibrosis with little or no modification. The improved survival and quality of life after transplantation have provided encouragement to extend this treatment to an increasing number of recipients. As the demands have increased and the supply of suitable donor organs has shown little change careful regulation of these scarce resources has become crucial. This can be achieved only by careful selection of patients and management by an experienced transplant team. Although this provides hope for countless patients and their carers not all patients will survive to benefit from transplantation. Heroic measures to prolong life are no more appropriate for the terminally ill patient with cystic fibrosis than they were 10 years ago. Early referral, with vigorous attention to preservation of respiratory function and nutrition, of stable patients awaiting surgery increases the likelihood of their surviving until they can receive a transplant-and afterwards.

1 British Paediatric Association Working Party on Cystic Fibrosis. Cystic fibrosis in the United Kingdom 1977-85 an improving picture. $B M J$ 1988;297:1599-602.

2 Jones K, Higenbottam T, Wallwork J. Successful heart-lung transplantation for cystic fibrosis. Chest 1988;93:644-5.

3 Scott J, Higenbottam T, Hutter J, et al. Heart-lung transplantation for cystic fibrosis. Lancet 1988;ii:192-4.

4 Reitz BA, Burton NA, Jamieson SW, et al. Autotransplantation and allotransplantation in primates with extended survival. J Thorac Cardiovasc Surg 1980;80:360-72.

5 Reitz BA, Wallwork JL, Hunt SA, et al. Heart-lung transplantation. Successful therapy for patients with pulmonary vascular disease. $N$ Engl J Med 1982;306:557-64.

6 Fradet G, Smyth RL, Scott JP, et al. Cystic fibrosis: a new challenge for cardiothoracic surgery. Eur $J$ Cardiothorac Surg 1990 (in press)

7 Cooper JD, Patterson GA, Grossman R, et al. Double-lung transplant for advanced chronic obstructive lung disease. Am Rev Respir Dis 1989;139:303-7.

8 Patterson GA. Double lung transplantation. Pediatr Pulmonol 1989;suppl 4:56-7.

9 Burke CM, Theodore J, Dawkins KD, et al. Post-operative obliterative bronchiolitis and other late lung sequelae in human heart-lung transplantation. Chest 1984;86:824-9.

10 Yousem SA, Paradis IL, Dauber JH, et al. Pulmonary arteriosclerosis in long-term human heart-lung transplan recipients. Transplantation 1989;47:564-9.

11 Metras D, Noirclerc M, Vaillant A, Brunet CH, Kreitman B. Double lung transplant. The role of bilateral bronchial suture. Transplant Proc 1990;22:1477-8.

12 Bishop JM, Cross KW. Physiological variables and mortality in patients with various categories of chronic respiratory disease. Bull Eur Physiopathol Respir 1984 20:495-500.

13 Siassi B, Moss AJ, Dooley RR. Clinical recognition of cor pulmonale in cystic fibrosis. J Pediatr 1971;78:794-805.

14 Stern RC, Borkat G, Hirschfeld SS, et al. Heart failure in cystic fibrosis. Treatment and prognosis of cor pulmonale with failure of the right side of the heart. Am J Dis Child 1980;134:267-72.

15 Huang NN, Schidlow DV, Szatrowski TH, et al. Clinica features, survival rate and prognostic factors in youn adults with cystic fibrosis. Am J Med 1987;82:871-9.

16 Smyth RL, Scott JP, Higenbottam TW, et al. Assessment for heart-lung transplantation in adults with cystic fibrosis [abstract]. Pediatr Pulmonol 1989;suppl 4:143.

17 Smyth RL, Scott JP, McGoldrick JP, Higenbottam TW, Wallwork J. Heart-lung transplantation for pneumothorax in cystic fibrosis. Ann Thorac Surg 1989;48:744-5

18 Nagel RA, Westaby D, Javaid A, et al. Liver disease and bileduct abnormalities in adults with cystic fibrosis. Lancet 1989;ii:1422-5.

19 Wallwork J, Williams R, Calne RY. Transplantation of liver, heart and lungs for primary biliary cirrhosis and primary pulmonary hypertension. Lancet 1987;ii:182-5.

20 Hutter JA, Despins P, Higenbottam T, Stewart S, Wallwork J. Heart-lung transplantation: better use of resources. $A m$ J Med 1988;85:4-11.

21 Caine N, Higenbottam TW, Wallwork J. Prospective study comparing quality of life before and after heart-lun transplantation. Transplant Proc (in press).

22 Smyth RL, Higenbottam TW, Scott JP, Wallwork J. Transplantation of the lungs. Respir Med 1989;83:459-66. 23 Higenbottam T, Stewart S, Penketh A, Wallwork J. Trans- 
bronchial biopsy for the diagnosis of rejection in heartlung transplant recipients. Transplantation 1988;46: 532-9.

24 Penketh AR, Higenbottam TW, Hutter J, Coutts C, Stewart $S$, Wallwork J. Clinical experience in the management of pulmonary opportunistic infection and rejection in heartlung transplant recipients. Thorax 1988;43:762-9.

25 Scott JP, Higenbottam TW, Clelland C, et al. The natural history of obliterative bronchiolitis in heart-lung transplant recipients. $J$ Heart Transplant (in press).

26 Jeffrey I, Durrans D, Wells $M$, Fox $H$. The pathology of meconium ileus equivalent. J Clin Pathol 1983;36:1292-7.

27 Smyth RL, Scott JP, Higenbottam TW, et al. The use of heart-lung transplantation in the management of terminal pulmonary complications of cystic fibrosis. Transplant Proc (in press)

28 Smyth RL, Scott JP, McGoldrick JP, Wallwork J, Higenbottam TW. Successful use of transbronchial biopsies in a patient with multiple opportunistic infection.
Respir Med 1989;83:505-7.

29 Scott JP, Smyth RL, McGoldrick JP, Higenbottam TW, Wallwork J. Cyclosporine dosing in cystic fibrosis patients following heart-lung transplantation. Transplantation 1989;48:744-5.

30 Knowles M, Gatzy J, Boucher R. Increased bioelectric potential difference across respiratory epithelia in cystic fotential difference across respiratory ep

31 Wood A, Higenbottam T, Jackson M, Scott J, Stewart S, Wallwork J. Airway mucosal bioelectric potential difference in cystic fibrosis after lung transplantation. $A m R e v$ Respir Dis 1989;140:1645-9.

32 Yankaskas JR, Knowles MR, Gatzy JT, Boucher RC. Persistence of abnormal chloride ion permeability in cystic fibrosis nasal epithelial cells in heterologous culture. Lancet 1985;i:954-6.

33 Dummer JS, Montero CG, Griffith BP, Hardesty RL, Paradis IL, Ho M. Infections in heart-lung transplant recipients. Transplantation 1986;41:725-9. 